\title{
On the Limitation of Ultra-Wideband Technique for Medical Scanning Systems
}

\author{
Siamak Sarjoghian, Yasir Alfadhl, and Xiaodong Chen \\ School of Electronic Engineering and Computer Science \\ Queen Mary University of London \\ London E1 4NS, UK \\ s.sarjoghian@qmul.ac.uk
}

\begin{abstract}
In this study, the ultra-wideband (UWB) technique and its capability to detect the cells and layer of a human organs in medical scanning applications has been considered. Advantages and disadvantages of the technique has been highlighted with respect to the capability and accuracy of a medical scanning system especially those using the pulse reflected-based technique. The study contrasts the difference between advantages of using UWB techniques for medical applications, such as eliminating ionising effects, and disadvantages, mainly due to those associated with penetration depth, range resolution, and cost within the unlicensed UWB frequency range (3.1-10.6 GHz). The above disadvantageous factors can cause drawbacks which limit the application of such techniques in human tissue scanning systems for early stages diagnostics. The UWB system has been studied well for the centre frequencies of $6 \mathrm{GHz}$ and $4 \mathrm{GHz}$, which found to have more potential in the UWB region among the researchers, however the outcomes indicates that there was a lack of knowledge base on the limitations associated with each of these frequencies.
\end{abstract}

Keywords—penetration depth; range resolution; $U W B$.

\section{INTRODUCTION}

UWB can provide promising advantages in using waves for transferring the information over bandwidths of larger than 500$\mathrm{MHz}$. Radio technology is originally aimed for short-range and indoor wireless communications with low power consumption. It utilises a large portion of spectrum to improve the speed and to reduce the interference of data transfer, compared to other existing technologies. The independent governmental regulator and competition authority for the UK Office of Communications (Ofcom) and the Federal Communications Commission (FCC) regulation of the UWB in the US allocates the regulation for the UWB that allows the unlicensed operating within the range of 3.1 to $10.6 \mathrm{GHz}$, and $41.3 \mathrm{dBm} / \mathrm{MHz}$ transmitted power [1].

In this technique, the signals diffuse across a wide range of frequencies in order to detect a subject accurately. It has high data rate in comparison to the other wireless technologies that enable this method to send trains of signal pulses contains of hundreds of millions pulses per second. As it is small and very low-power it fits the requirement for use as indoor applications. In the last few years the UWB has been expressed as a potential candidate for medical purposes compared to the other scanning technologies due to its unique properties, such as:

High data rates: The large bandwidth occupied by the UWB $(<500 \mathrm{MHz})$ gives potential of speeds over $500 \mathrm{Mb} / \mathrm{s}$ and higher speed over shorter distances.
Low cost: UWB is a low power technology, therefore low cost technique where the capability to frankly adjust a pulse onto an antenna is possibly the same as a simple transmitter that make the transceiver enormously cheaper to manufacture.

Multipath immunity: UWB pulses can supply a high resolution of reflected pulses at the receiver due to the narrow shape pulses, which cause by its capability of pulse separation. This is essential in channels due to pulses interfering each other are the major complication to error-free wireless systems.

Ranging and communication at the same time: Using UWB technique provides user both precise ranging (object location) and high-speed data communication in the same device presents intriguing possibilities for new applications.

Eliminating ionising effect: Ionising radiation is the main concern of the scanning technologies, the UWB is identify as a good candidate for scanning as it can eliminate ionising effect compared with other RF techniques currently being used [2].

With respect to the fact that each technique has some unique properties as well as limitations, this study tries to highlight the fact that however there are lots of researches had been conducted in the past in order to use the technique for medical applications such as human abdominal fat, breast cancer, skin cancer, and blood cancer detection regarding to capability of the technique. But still none of the works have been successfully manufactured due to the lack of accuracy and the cost compared to the other techniques being used by the health professionals [3-6].

An UWB technique generally categorised by the pulse is being used by the system; there are different types of UWB pulses such as simple Gaussian and the derivative pulses shape or more complex orthogonal pulses, such as Hermit pulses. Furthermore, in order to select a specific pulse for a system, there are parameters that have to be taken into the account:

Pulse shape: Each UWB pulse has its own shape that can be used for different purposes base on the application such as data rate and resolution.

Centre frequency: The pulse centre frequency is important, as it can allocate the penetration depth of pulse through medium, this means by lowering the centre frequency, the capability of the pulse to penetrate into the medium will increases.

Bandwidth: Other pulse standard that is essential to identify for the system is pulse bandwidth; this parameter can verify the system resolution as the pulse bandwidth will increase, the width 
of the pulse will reduce and therefore, the pulse can detect the smaller object due to its short width.

The next important factor in any UWB medical scanning system is the scanning medium, which changes refer to different part of the human body, such as abdominal, breast, skin, and blood. Each of these medium has their own tissue structures and dielectric properties in a specific frequency.

The tissue types are essentially treated as dielectrics and therefore their material properties are characterised by relative permittivity $(\varepsilon)$ and conductivity $(\sigma)$. The complex relative permittivity is defined as in equation (1):

$$
\varepsilon=\varepsilon^{\prime}-j \varepsilon^{\prime \prime} .
$$

where, $\varepsilon^{\prime}$ is the real part of the relative permittivity, and $\varepsilon^{\prime \prime}$ is the imaginary part or the loss factor, and is given by (2) as:

$$
\varepsilon^{\prime \prime}=\frac{\sigma}{\varepsilon_{0} \omega} .
$$

The reaction of any material to the electric field comprises of a physical shift of charge, and the kinetics of the shift which varies with frequency and gives rise to the material properties. This reaction can be modelled in terms of resonance and relaxation. The Debye model is used in order to characterise the conductivity in terms of a relaxation time $(\tau)$. The first and the second order of Debye equations are given in equations (3-4), which are used to analyse the dielectric properties of biological tissue in various frequencies [7].

$$
\begin{gathered}
\varepsilon(\omega)=\varepsilon_{\infty}+\frac{\varepsilon_{s}-\varepsilon_{\infty}}{1+j \omega \tau} . \\
\varepsilon(\omega)=\varepsilon_{\infty}+\frac{\varepsilon_{s 1}-\varepsilon_{\infty}}{1+j \omega \tau_{1}}+\frac{\varepsilon_{s 2}-\varepsilon_{\infty}}{1+j \omega \tau_{2}} .
\end{gathered}
$$

where $\varepsilon(\omega)$ is the complex permittivity, $\varepsilon_{s}$ is relative static permittivity at low frequencies, and $\varepsilon_{\infty}$ is the relative optical permittivity at high frequencies.

Table 1 shows the dielectric properties and penetration depth of the particular human tissues types that is being used for the monitoring systems which is already mentioned within the 6 GHz. The centre frequency is chosen based on its advantage of covering the highest bandwidth which can offer ideal range resolution for the system; however, as it is depicted in table 1, it does not provide considerable penetration depth for the system penetrating inside human body which is vital; bear in mind by shifting down the frequency for more penetration, the bandwidth and consequently the range resolution will be reduced [8]

Table 1. Dielectric properties of the tissues in frequency of $6 \mathrm{GHz}$ [8-9].

\begin{tabular}{|c|c|c|c|c|}
\hline $\begin{array}{c}\text { Tissue } \\
\text { Name }\end{array}$ & $\begin{array}{c}\text { Frequency } \\
\text { (GHz) }\end{array}$ & $\begin{array}{c}\text { Conductivity } \\
(\mathbf{S} / \mathbf{m})\end{array}$ & $\begin{array}{c}\text { Relative } \\
\text { Permittivity }\end{array}$ & $\begin{array}{c}\text { Penetration } \\
\text { Depth } \\
(\mathbf{m m})\end{array}$ \\
\hline Skin (dry) & 6 & 3.89 & 34.9 & 8 \\
\hline Breast Fat & 6 & 0.44 & 4.46 & 26 \\
\hline Fat & 6 & 0.87 & 9.8 & 39 \\
\hline Muscle & 6 & 5.2 & 48.2 & 7 \\
\hline Blood & 6 & 6.8 & 52.2 & 6 \\
\hline Glandular & 6 & 4.5 & 28 & 6 \\
\hline $\begin{array}{c}\text { Cancerous } \\
\text { cells }\end{array}$ & 6 & 7.5 & 45 & 6 \\
\hline
\end{tabular}

\section{UWB PULSE CAPABILITY}

\section{A. Penetration Depth}

The propagation constant of each model layer can be defined as $(\gamma=\alpha+j \beta)$, where $\alpha$ is the attenuation constant, $\beta$ is the phase constant, and $\delta$ is the skin depth constant which can be defined by equations (5-7), respectively:

$$
\begin{aligned}
& \alpha=\omega \sqrt{\mu_{0} \varepsilon_{0} \varepsilon_{r}}\left[\frac{1}{2}\left(\sqrt{1+\left(\frac{\sigma}{\omega \varepsilon_{0} \varepsilon_{r}}\right)^{2}}-1\right)\right]^{\frac{1}{2}}(N P / m) . \\
& \beta=\omega \sqrt{\mu_{0} \varepsilon_{0} \varepsilon_{r}}\left[\frac{1}{2}\left(\sqrt{1+\left(\frac{\sigma}{\omega \varepsilon_{0} \varepsilon_{r}}\right)^{2}}+1\right)\right]^{\frac{1}{2}}(\mathrm{rad} / \mathrm{m}) . \\
& \delta=\frac{1}{\alpha}=\frac{1}{\omega \sqrt{\mu_{0} \varepsilon_{0} \varepsilon_{r}}\left[\frac{1}{2}\left(\sqrt{1+\left(\frac{\sigma}{\omega \varepsilon_{0} \varepsilon_{r}}\right)^{2}}+1\right)\right]^{\frac{1}{2}}}(\mathrm{~m}) .
\end{aligned}
$$

where $\sigma$ is the conductivity; $\omega=2 \pi \mathrm{f}$ is the angular frequency, $\varepsilon_{0}$ is the permittivity of free space, and $\mu_{0}$ is the permeability of free space. In considering the frequency dependent penetration depth of each tissue type, a pulse centre frequency of $\sim 6 \mathrm{GHz}$ is considered as the optimum value with regards to high achievable resolution that motivates researchers to employ in cases where penetration inside the tissue is not of the main concern [10-11].

Table 1, presents the penetration depth in $6 \mathrm{GHz}$ inside the popular tissue types, which is being used in most of the medical applications. The result demonstrates that the penetration depth in this frequency is not suitable for the medical scanning systems in which detection of layers or objects inside the body is of the main system objective.

For this reason, the researchers try to use a lower centre frequency for the systems in which the penetration inside the human body is of importance. On the other hand, by lowering the centre frequency, the pulse bandwidth will be reduced and consequently the resolution will be decreased. The resolution is a vital factor in health monitoring equipment, specifically those detecting cancer cells within the body, in which the treatment initiation at early stages are of crucial importance in the human life survival scenarios.

\section{B. Range Resolution}

Another important factor at any stationary radar system, which has to detect the different targets that are bound to each other, is range resolution (spatial resolution). Range resolution defines as the capability of any radar in order to differentiate between two or more targets that are very close to each other on the same bearing, but at different ranges. The degree of range resolution is subject to the width of the transmitted pulse, based on the type and size of targets, and the efficiency of the receiver.

In the $19^{\text {th }}$ century, the physicist John William Strutt, 3rd Baron Rayleigh produced a criterion that later named Rayleigh criterion, which identifies that for the purpose of detecting two targets in the same bearing, the crossing of two reflected scattering should be $9 \mathrm{~dB}$ below their maximum value, as also shown in Fig. 1. 


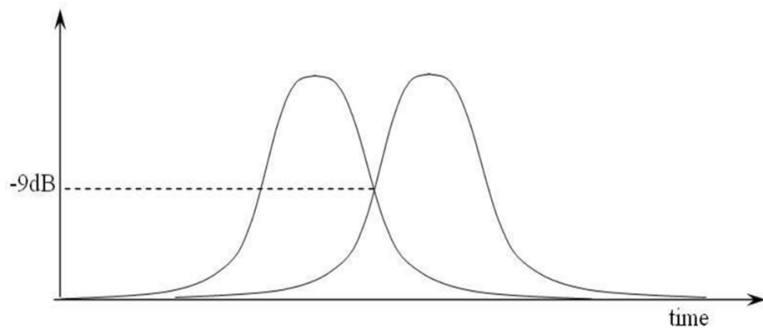

Fig. 1. Rayleigh criterion: limit of resolution for two point scatters [12]

Table 2. Tissue thickness and its required bandwidth for the appropriate range resolution.

\begin{tabular}{|c|c|c|}
\hline Tissue thickness (mm) & $\begin{array}{c}\text { Bandwidth } \\
B \frac{c}{2 \Delta R \sqrt{\varepsilon_{r}}}(G H z) \\
\text { free space }\end{array}$ & $\begin{array}{c}\text { Bandwidth } \\
B \frac{c}{2 \Delta R \sqrt{\varepsilon_{r}}}(\mathrm{GHz}) \\
\text { breast fat }\end{array}$ \\
\hline 2 & 75 & 35.6 \\
\hline 9.5 & 15.8 & 7.5 \\
\hline 20 & 7.5 & 3.5 \\
\hline 35 & 4.3 & $\mathbf{2}$ \\
\hline 75 & 2 & 0.94 \\
\hline
\end{tabular}

The pulse width is the key factor in the range resolution. A radar system, with all other elements at maximum efficiency, should be able to distinguish targets separated by one-half of the pulse width time; hence, the theoretical range resolution of a radar system can be defined as in equation (8):

$$
\stackrel{\text { yields }}{\longrightarrow} \Delta R=R_{n-1}-R_{n}=\frac{c . \tau}{2 \sqrt{\varepsilon_{r}}}(m) .
$$

where $c$ represents speed of light in free space, $\tau(\mathrm{s})$ is the pulse width in second and $\Delta \mathrm{R}(\mathrm{m})$ is the distance between two targets in meter. This means by reducing the pulse width and hence increasing the pulse bandwidth, smallest target can be resolved by the system due to eliminating the problem of overlapping of the reflected pulses. In the case that is a limitation for decreasing the pulse width due to the sampling and down conversion issues that are introduced for the system design, a pulse compression technique can be employed to increase the pulse bandwidth. The range resolution of the radar is given by the bandwidth of the transmitted pulse (B), instead of its pulse width [i.e., depicted in the equation (9)]. This allows very high resolution to be obtained with long pulses, thus resulting in a higher average power [12].

$$
\Delta R \geq \frac{c}{2 B \sqrt{\varepsilon_{r}}}(m) \stackrel{\text { yields }}{\longrightarrow} B \geq \frac{c}{2 \Delta R \sqrt{\varepsilon_{r}}}
$$

The required bandwidth for any tissue type with a different thickness has been calculated, and presented in Table 2, which indicates that for detection of $2 \mathrm{~mm}$ tissue layer, such as skin in free space, there is a need of pulse with a bandwidth of higher than $75 \mathrm{GHz}$, which is not achievable using the UWB pulses, however, this can be reduced in cases where detection of object within another medium, such as breast fat in the breast cancer detection, is of the main aim. The pulse with a centre frequency of $6 \mathrm{GHz}$, which provides up to the maximum bandwidth of 7.5 $\mathrm{GHz}$, is only capable of detecting a tumour bigger than $10 \mathrm{~mm}$, where there is not enough penetration, and finally, using pulses with $1 \mathrm{GHz}$ of bandwidth, the system is then capable to detect the tumour bigger than $75 \mathrm{~mm}$ in breast fat medium; however, with respect to the penetration depth defined in the Table 1, there will not be a good penetration depth in this centre frequency. Furthermore, with respect to the processing such data, the cost of hardware will be increased (i.e., due to large bandwidth), such as FPGAs, ADCs, and VCOs, along with other required devices. With respect to the outcomes that have been already presented in Table 1, in the case in which there is not an existence of high permittivity medium, such as a female breast, the penetration depth should be reduced to minimum of $4 \mathrm{GHz}$, to be able to penetrate inside the breast. However, with reducing the centre frequency to $4 \mathrm{GHz}$, the pulse bandwidth will be reduced to around $2 \mathrm{GHz}$, in which the detection of objects with minimum size of $35 \mathrm{~mm}$ is then possible in the breast fat medium. This can prove that the system unreliability for medical applications, such as cancer detection and specifically the reflected-based systems in which the early detection is a vital survival issue. There are some processing techniques that are being developed and further used in the previous researches, to reduce the cost of the overall system that has its own limitations, according to the following:

\section{Processing Technique}

Based on the previous research, in order to detect the small object such as human tissues, which is more likely to have a very thin layers, such as skin $(2 \mathrm{~mm})$, there is needs to design a radar with a wide bandwidth in order to improve the range resolution of the system, to be able to distinguish the reflected pulse from each layer. As it is mentioned, in order to increase the bandwidth and therefore range resolution, there are some drawbacks will be built into the system, namely less penetration depth, and more increase in hardware cost, because of the necessities of essential sampling and processing hardware. However, there are a number of algorithms that have been developed that can be used in order to increase the range resolution of the reflected pulse, without extending the pulse bandwidth. But, these algorithms exhibit limitations in terms of the complexity and cost. Many of these methods, which are defined as super-resolution techniques, are developed in the past. The main algorithms can be summarised as the maximum entropy method (i.e., Burg), covariance method (i.e., Prony), CLEAN, matrix pencil, ESPRIT, and MUSIC.

In 1997, the Lincoln Laboratory has established a method for the UWB radar signature of an object by using sparse sub-band measurements. This method expands the range resolution of the reflected pulses by increasing operative bandwidth based on the bandwidth extrapolation algorithm (BWE) [13-14]. The BWE algorithm intends to expand the effective bandwidth of radars by prediction of the object response at specific frequencies that lie outside the measurement bands. The purpose technique intends to increase the range resolution by the factor of two to three that can improve the quality of images dramatically. However, the technique improves the resolution of the image developed by the system, the method introduces some limitation, such as [15]:

1. The algorithm works primarily on signal processing models which characterises a complex target, as collection of point scattering along with the frequency independent amplitude.

2. The method is satisfactory for wideband signal processing in which the waveforms have a small fractional bandwidth, according to the centre frequency, compared with the radar; however, the scattering amplitude of the discrete scattering centres can be fluctuated significantly with the frequency. 


\section{DISCCUSION}

The defined parameters which have been well-explained in the previous section of this paper are in good agreement with the UWB limitation based on following issues:

With respect to the penetration depth, which mainly depends on the application, if there is a need for the pulse to be penetrated inside the body, which in most cases, is a crucial factor, there is a need to employ the centre frequency lower than the frequencies provided by the unlicensed UWB method (3.1-10.6 GHz). This gives rise to the first alert whether the technique is an appropriate alternative for the human body scanning systems.

Also, with respect to the range resolution, and based on the analyses presented throughout the paper for the layers or objects detection in the body, the results demonstrated that for detecting the object with thickness of $2 \mathrm{~mm}$, there is a need for the pulse with the bandwidth of higher than $75 \mathrm{GHz}$ in free space and 35.6 $\mathrm{GHz}$ in breast fat medium. This rises the second alert whether the technique with the intended maximum bandwidth of 7.5 $\mathrm{GHz}$, is capable of detecting objects smaller than $10 \mathrm{~mm}$ in any medium, especially in the case that penetration depth is another important factor, and has to be further satisfied by lowering the centre frequency, which is the result of further reduction in the bandwidth, and consequently resolution decrease.

The cost concern is also another major limitation that should be considered during the implementation, with respect to the hardware components (e.g., ADC and FPGA) needed to process such a wide UWB pulse. It is also worth mentioning that the cost can be reduced using the signal processing algorithms; however, this would result in the system resolution decrease, due to the estimation, along with the complexity increase, due to the timeconsuming data processing based on these methods.

\section{CONCLUSION}

The results demonstrate that the unlicensed UWB spectrum (i.e., 3.1-10.6 GHz) is not a promising technology for certain medical applications, based on the analysed system parameters including penetration depth, resolution, and cost; as the important factors for the intended system design and deployment. However, there are still a number of researches being carried out to conduct the feasibility analysis of the method employment for the medical applications, but as it has been presented in this investigation, the bandwidth and centre frequency, as the major performance factors, are not being considered for the appropriate design and implementation based on the UWB technology.

\section{REFERENCES}

[1] F. C. Commission, "Revision of part 15 of the commission's rules regarding ultra-wideband transmission systems, FIRST REPORT AND ORDER," ET Docket 98-153, FCC 02-48, pp. 100-118, 2002.

[2] M. Ghavami, L. Michael, and R. Kohno, Ultra Wideband Signals and Systems in Communication Engineering, $2^{\text {nd }}$ Edition, Wiley, 2007.

[3] S. Sarjoghian et al., "A novel wide-band reflection-based system for measuring abdominal fat in humans" in URSI Commission B International Symposium on Electromagnetic Theory (EMTS), Aug. 2016.

[4] M. Klemm et al., "Experimental and clinical results of breast cancer detection using UWB microwave radar" in IEEE Antennas and Propagation Society International Symposium, Jul. 2008.

[5] M. A. A. Eldosoky, "The ability of ultra wideband signals in detection of the skin tumour" in URSI Assembly and Scientific Symp., 2008.

[6] M. A. A. Eldosoky and H. M. Moustafa, "Detection of the blood Leukemia by using the ultra wide band pulses" in XXXth URSI General Assembly and Scientific Symposium, Aug. 2011.

[7] Y. Alfadhl, Numerical evaluations on the interaction of electromagnetic fields with animals and biological tissues, Ph.D. Thesis, Queen Mary University of London, 2005.

[8] T. Sugitani et al., "Complex permittivities of breast tumor tissues obtained from cancer surgeries" in Applied Physics Letters, June 2014.

[9] D. Andreuccetti and R. Fossi, "Dielectric properties of human tissues definitions, parametric model, and computer codes," Report N.TR/ICEMM/13.00, IFAC-CNR, Firenze (I), Sep. 2000.

[10] G. Kang and O. P. Gandh, "Effect of dielectric properties on the peak 1and 10-g SAR for $802.11 \mathrm{a} / \mathrm{b} / \mathrm{g}$ frequencies 2.45 and 5.15 to $5.85 \mathrm{GHz}$," IEEE Trans. Electromagnetic Compatibility, vol. 46, no. 2, May 2004.

[11] A. Peyman et al., "Dielectric properties of tissues at microwave frequencies" MTHR Co-ordination Team UK, Mar. 2005.

[12] S. Kingsley and S. Quegan, Understanding Radar Systems, SciTech Publishing, 1999.

[13] K. M. Cuomo, "A bandwidth extrapolation technique for improved range resolution of coherent radar data," Project Report CJP-60 Rev.1, Lincoln Laboratory, Dec. 1992.

[14] S. L. Borison et al., "Super-resolution methods for wideband radar," The Lincoln Laboratory Journal, vol. 5, no. 3, pp. 441-461, 1992.

[15] K. M. Cuomo et al., "Ultra-wideband coherent processing" The Lincoln Laboratory Journal, vol. 10, no. 2, pp. 203-222, 1997. 